\title{
A Brief Review on Combining Left and Right Palmprint Image for More Accurate Personal Identification
}

\author{
Pravin P. Kalyankar \\ Pursuing PhD at \\ Dr. B.A.M.U. Aurangabad
}

\author{
Bhagyashri K. Mane \\ Pursuing ME at \\ Terna College of Engg. Osmanabad
}

\begin{abstract}
In this paper, an effective biometrics method based on hand geometry is presented for biometric identification or verification system. Biometrics-based authentication is a verification approach using the biological features inherent in each individual. They are processed based on the identical, portable, and arduous duplicate characteristics. The principal lines and texture are two kinds of salient features of palmprint. A few years ago, a new branch of biometrics technology, palmprint authentication was proposed whereby line and points are extracted from palms for personal identification. In this paper, we consider palmprint as a piece of lines and texture and apply palmprint identification techniques for extracting feature to palmprint authentication.
\end{abstract}

\section{General Terms}

Biometrics, Palmprint identification, multibiometrics etc.

\section{Keywords}

Palmprint identification method, feature extraction, palmprint.

\section{INTRODUCTION}

Biometric identification is an emerging technology that can solve security related problem in our networked society. Recently, biometric features have been widely used in many personal identification application. Palmprint attracted much attention. The palmprint contains not only principle curves and wrinkles but also rich texture and miniscule points, so the palmprint identification is able to achieve a high accuracy because of available rich information in palmprint. Automatic personal identification using biometric information is playing an important role in the applications of public security, access control, forensic, e-banking, etc. Many kinds of biometric authentication techniques.

Palmprint based biometric approaches have been intensively developed, Because they possess several advantages over other systems. Palmprint a large inner surface on our hand contains many lines features. For example: principle lines, wrinkles and ridges. Because of the large surface and rich lines features, we expect palm prints to be robust to noise and to have high in individuality. Palm print is preferred compared to other methods such as fingerprint or iris because it is distinctive, easily captured by low resolution devices as well

as contains additional features such as principal line [9]. With the help of palm geometry, a highly accurate biometric system can be designed. Iris input devices are expensive and the method is intrusive as people might fear of adverse effects on their eyes. Fingerprint identification requires high resolution capturing devices and may not be suitable for all as some may be finger deficient.

\section{PALMPRINT IDENTIFICATION METHODS}

There are various palmprint identification method works on individual biometric feature such as line, palmcode, palmvision, curve, texture and points etc.

\subsection{A Line Based Method}

Lines are the basic feature of palmprint and line based methodes play an important role in palmprint verification and identification. It is well known that palm lines consist of wrinkles and principal lines. And principal lines can be treated as a separate feature to characterize a palm. Therefore, there are several reasons to carefully study principal lines based approaches. At first, principal lines based approaches can be jointly considered with the person's habit. For instance, when human beings are comparing two palmprints, they instinctively compare principal lines. Secondly, principal lines are generally more stable than wrinkles. The latter is easily masked by bad illumination condition, compression, and noise. Thirdly, principal lines can act as an important component in multiple features based approaches. Fourthly, in some special cases, for example, when the police is searching for some palmprints with similar principal lines, other features cannot be used to replace principal lines. At last, principal lines can be used in palmprint classification or fast retrieval schemes.

In general,most palms have three principal lines: the heartline, headline, and lifeline, which are the longest and widest lines in the palmprint image and have stable line shapes and positions shown in following figure 1. (see Fig. 1(a)) In addition, a lot of palmprints may have more or less principal lines due to their diversity and complexity (see Fig. $1(b))$.

In this paper, assuming that within a few of palmprints one or two longest and strongest wrinkles that have similar directions to three principal lines are also regarded as a part of principal lines (see Fig. 1(c)). a

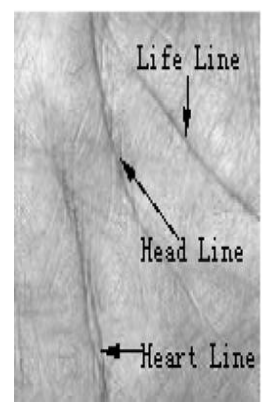

b

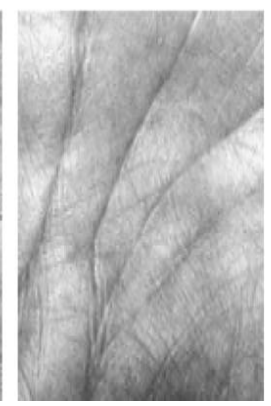

C

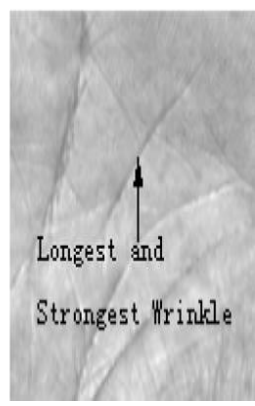

Fig 1:Three typical palmprint images. 
In line based method to extracting the palmprint lines use for lines or edge detectors. It is most usefull in palmprint identification and verification. Palmprint principal lines can be extracted by using the Following operation.
a. Gobor filter
b. Sobel operation
c. Morphological operation

The Gabor filter is also a powerful tool to detect the directional energies of lines. For example, it was often used for fingerprint image enhancement.. However, the Gabor filter is not suitable for detecting the directional energies of palm lines. The main reason is that its band limitation restricts its ability to detect the lines with different widths.

The sobel operation performs a 2D spatial gradient measurement on an image and so emphasizes regions of high spatial frequency that correstond to edgs. It is used to find the approximate absolute gradient magnitude at each point in an input grayscale image.

In morphological operation the number of pixel added or removed from the object in an image depends on the size and shape of the structuring elements used to process the image. There are two way of morphological Dilation and Erosion Morphology is a broad set of image processing operation that process image based on shapes. Han et al. proposed using Sobel and morphological operations to extract the linelike features from palmprint images obtained using a scanner

\subsection{Coding Based Method}

The most influential palmprint identification method is the Coding based method. Representative coding based, competitive code method, ordinal code method, palmcode method and Binary Orientation Co-occurrence Vector(BOCV)[4] method. Coding based method, which encode the response of a bank of filters and have been very successful in palmprint representation. After convolving the palmprint image with 2D Gabor filter, The palm code method encode the phase of the filter responses as binary features. To reduce the spatial correlation of palm code, Fusion code method introduces encode the phase of the filter response whose magnitude is maximum. The competitive code, the ordinal code and the Robust line orientation code(RLOC) are Orientation coding method which involves three component:

the filter bank design for extracting the palmprint orientation information,the coding scheme for and efficient representation of orientation information, and the matching approach for fast and accurate palmprint recognition. A binary orientation cooccurrence vector[BOCV] method to represent multiple orientation for a local resion.

\subsection{Representation Based Method}

All representation based methods can be easily applied to perform paimprint identification. The representation based method uses training samples to represent the test sample, and selects a candidate class with the maximum contribution to the sample. The collaborative Representation based Classification [CRC] method, Sparse Representation-Based Classification [SRC] method and Two-PhaseTest Sample Sparse Representation [TPTSSR] [1] method are two representative representation based methods. Almost all representation based methods can be easily applied to perform palmprint identification.

\subsection{SIFT Based Method}

SIFT was originally proposed in [8] for object classification application, which are introduced for contactless palmprint identification in recent years.Because the contactless palmprint images have severe variations in poses,scales,rotation and translation and scale variation and hence promising to solve the deformation problem. Which make conventional palmprint feature extraction methods on contactless imaging schemes questionable and therefore, the identification accuracy of conventional palmprint recognition methods is usually not satisfactory for contactless palmprint identification. The features extracted by SIFT are invariant to image scaling, rotation and partially invariant to the change of projection and illumination. Therefore, the SIFT based method is insensitive to the scaling, rotation, projective and illumination factors, and thus is advisable for the contactless palmprint identification. The SIFT based method firstly searches over all scales and image locations by using a difference-of-Gaussian function to identify potential interest points. Then an elaborated model is used to determine finer location and scale at each candidate location and keypoints are selected based on the stability. Then one or more orientations are assigned to each keypoint location based on local image gradient directions. Finally, the local image gradients are evaluated at the selected scale in the region around each keypoint.

\subsubsection{Sift Based Palmprint Recognition} Considering Single Palmprint

Many times when images goes under certain transformation deletion of pegs or other auxiliary schemas on image acquisition devices can unavoidably introduce variations of palm print images due to hand unsteadiness. In such cases, this method is used to solve the problem, this method proposes a palm print image alignment method based on SIFT features [13]. SIFT (Scale Invariant feature transform) features are based on local information, which are invariant to image shift, scale, and rotation variations, and partially invariant to illumination and projective changes. In SIFT based algorithm, image is first preprocessed using Gabor filter [13]. After preprocessing the image is then for SIFT feature extraction where Scale space construction is performed followed by Key point localization. Orientation assignment is done which is then followed by Descriptor computing.

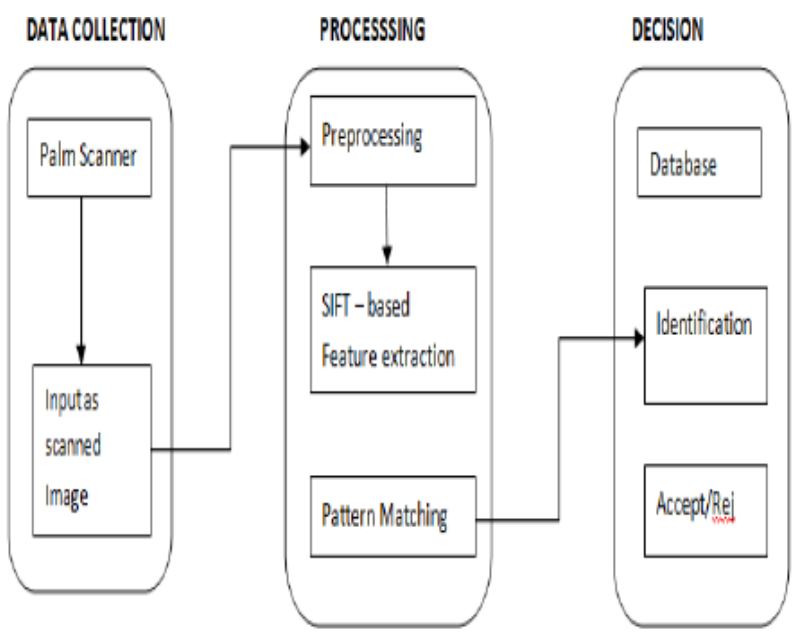

Fig 2: Working of SIFT method. 


\subsubsection{SIFT Algorithm Description}

Scale-invariant feature transform (or SIFT) is an algorithm in computer vision to detect and describe local features in images. It is an image descriptor for image-based matching and recognition. This descriptor as well as related image descriptors are used for a large number of purposes in computer vision related to point matching between different views of a 3-D scene and view-based object recognition. The SIFT descriptor is invariant to translations, rotations and scaling transformations in the image domain and robust to moderate perspective transformations and illumination variations. Experimentally, the SIFT descriptor has been proven to be very useful in practice for image matching and object recognition under real-world conditions. SIFT keypoints of objects are first extracted from a set of reference images and stored in a database. An object is recognized in a new image by individually comparing each feature from the new image to this database and finding candidate matching features based on Euclidean distance of their feature vectors. From the full set of matches, subsets of keypoints that agree on the object and its location, scale, and orientation in the new image are identified to filter out good matches. The determination of consistent clusters is performed rapidly by using an efficient hash table implementation of the generalized Hough transform.

Each cluster of 3 or more features that agree on an object and its pose is then subject to further detailed model verification and subsequently outliers are discarded. Finally the probability that a particular set of features indicates the presence of an object is computed, given the accuracy of fit and number of probable false matches.

\subsection{Subspace Based Methods}

Subspace based methods include the LDA and PCA. The key idea behind LDA is to find an optimal projection matrix $1 \mathrm{~V}$ and transforms the original space to a lower-dimensional feature space. LDA maximizes the Euclidean distance of samples from different classes and also minimizes the distance of samples from the same classes.PCA is use to find an orthogonal subspace that preserves the maximum variance of the original data.This method tries to find the best set of projection direction in the sample space that will maximize the total scatter across all samples. PCA choose the first few principal components and uses them to transform the samples in to a low-dimensional feature space.

\section{ACKNOWLEDGMENTS}

I would like to express my deep gratitude to my research, Prof. P. P. Kalyankar has a research guide, for their patient guidance, enthusiastic encouragement and useful critiques of this research work. I would also like to extend my thanks to the technicians of the laboratory of the Computer department for their help in offering me the resources in running the program.

\section{REFERENCES}

[1] X. Wu,Q. Zhao, and W. Bu, “ A SIFT based contactless palmprint verification approach using iterative RANSAC and local palmprint descriptors ", Pttern Recognition, vol.47, no.10,pp.3314-3326,oct.2014.

[2] Y. Xu,D. Zhang, J.Yang, and J-Y. YANG.” A two-phase test sample sparse representation method for use with face Recognition. " IEEE Trans Circuit Syst. Video technol, Vol. 21, N0.9 , PP. 1255-1262, Sep. 2011.

[3] FengYue, Wangmeng Zuoand Kuanquan Wang,"FCMbased orientation selection for competitive coding based "Palmprint recognition", Biocomputing Research, and school of computer science and technology, Harbin Institute of Technology, Harbin, China,2010".

[4] Z. Guo, D. Zhang, L. Zhang, and W. Zuo, “ Palmprint verification using binary orientation co-occurrence vector," Pattern Recognit Letts, vol.30, no.13,pp.1219. 1227.Oct.2099.

[5] D. S. Huang, W.Jia, and D. Zhang, "Palmprint verification based on principle lines"Pattern Recognir, vol.41, no.4 pp.1316-1328.Apr.2008

[6] Y. Hao,Z. Sun, and T.Tan, "Comparative studies on multispectral palm image fusion for biometrics ," In Proc. Asian Conf. Comput. Vis,pp.12-21,2007.

[7] R.K.Rowe, U.Uludag, M.Demirkus, S.Parthasaradhi, and A.K. Jain" A Multispectral whole-hand biometric authentication system," in Proc.Biometric Symp., Biometric Consortium Conf., Baltimore, MD, pp.16,2007 .

[8] D. G.Lowe, " Distinctive image feature from scaleinvariant keypoint.” Int.J. Comput. Vis..., vol.60, no. 2, pp.90-110.Nov.2004.

[9] A. W. K. Kong, D. Zhang, and M. S. Kamel, "A survey of palmprint recognition," Pattern Recognit., vol. 42, no. 7, pp. 1408-1418, Jul. 2009.

[10] D. Zhang, W. Zuo, and F. Yue, "A comparative study of palmprint recognition algorithms," ACM Comput. Surv. vol. 44, no. 1, pp. 1-37, Jan. 2012.

[11] Z. Sun, T. Tan, Y. Wang, and S. Z. Li, "Ordinal palmprint represention for personal identification [represention read representation]," in Proc. IEEE Conf. comput Vis. Pattern Recognit, Vol. 1. Jun. 2005, pp. 279-284. 